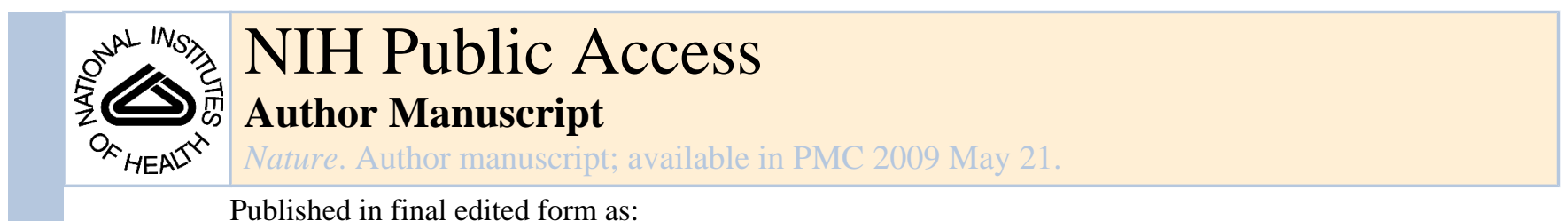

Published in final edited form as:

Nature. 2008 November 13; 456(7219): 183-185. doi:10.1038/456183a.

\title{
The voltage-sensor quartet
}

\author{
J. R. Bankston and R. S. Kass \\ Department of Pharmacology, College of Physicians and Surgeons, Columbia University, New York, \\ New York 10032, USA
}

\section{Abstract}

Decoding the workings of voltage-gated sodium channels is crucial because their mutation leads to severe disease and their activity is modulated by toxins and drugs. An innovative approach now allows such investigations.

Voltage-gated ion channels are pore-forming transmembrane proteins that open and close (gate) in response to changes in transmembrane voltage, enabling carefully controlled movement of ions across cell membranes. The precise gating of these channels underlies various biological phenomena, including the generation and propagation of electrical impulses in nerve and muscle cells, the secretion of hormones and neurotransmitters, and regulation of heart muscle contraction as well as of skeletal and vascular smooth muscles. On page 202 of this issue, Bosmans et al. ${ }^{1}$ deconstruct sodium channels to decipher the function of their four voltage sensors.

Sodium channels, like calcium, potassium and cyclic-nucleotide-gated channels, are composed of a tetramer of similar domains (I-IV), with each domain having six transmembrane segments. In both sodium and potassium channels, the segments within each domain can be divided into two basic units. The S5 and S6 segments of all four domains collectively constitute the pore, which allows rapid permeation of ions - more than 100 per second - across the cell membrane. By contrast, transmembrane segments S1-S4 of each domain act as voltage sensors. Within the S4 segments of each domain, positively charged amino-acid residues are interspersed with hydrophobic residues, and these positive charges play an essential part in voltage sensing ${ }^{2}$. Moreover, the structure of a potassium channel shows that $\mathrm{S} 4$ and the most extracellular part of S3 (S3b) form a voltage-sensing 'paddle', which moves across the membrane as a unit to open the channel and allow ions to flow ${ }^{3,4}$. Analysis of the functional roles of the voltage-sensing structures in sodium channels, however, has lagged behind, in part owing to the difficulty in studying these domains individually.

Bosmans and colleagues ${ }^{1}$ take an original approach to investigate the gating machinery of sodium channels. Inserting individual voltage-sensor paddle domains of two different sodium channels into a potassium channel, they found that, despite significant differences in sequence, the sodium-channel paddle motifs can function as part of a potassium channel (Fig. 1, overleaf). Thus, the authors could use potassium channels as functional templates for studying the behaviour of each of the four sodium-channel voltage sensors. They investigated two aspects of S4 structure/function: how these motifs respond to changes in membrane potential, and their unique binding sites for various toxins.

Each of the four voltage sensors of a sodium channel has specific kinetics and roles in channel gating. In response to depolarization of the cell membrane, voltage sensors of domains I-III 
move outwards to open the channel gate and so activate the channel. Inactivation follows when the domain-IV voltage sensor, whose movement lags behind the fast motion of the other three sensors, moves outwards and puts the channel in a non-conducting state that will not reopen during sustained membrane depolarization. For reasons that are not entirely understood, this paddle gets trapped in the channel-inactivating position. The slow recovery of this sensor after membrane repolarization potentially reflects the detachment of inactivation machinery, but could also simply reflect the intrinsic nature of this paddle and the environment through which it moves. Whether this voltage paddle also somehow contributes to activation is debated ${ }^{5}$.

Examining the kinetics of voltage-sensor movement in a chimaera of a potassium channel and the voltage paddle from domain IV of a sodium channel, Bosmans et al. show that slow movement of the sensor is simply an intrinsic property of this paddle, rather than a slowing of the paddle's motion by its association with the inactivation machinery. This intriguing observation is a first step towards a closer examination of the relationship between motion and inactivation of the domain-IV voltage sensor in sodium channels.

Toxins from various organisms, such as the tarantula and scorpion, target the voltage sensors of sodium channels, affecting the regulation of channel gating. Many of these toxins are also used as tools for examining sodium-channel gating, and understanding their precise site of action is important. So Bosmans et al. used their paddle-swapping approach to identify the binding sites of such toxins. They isolate the specific voltage sensors targeted by many of these toxins and draw two main conclusions.

First, interactions between toxins and the voltage sensors differ between sodium-channel subtypes. For instance, a toxin that interacts with domains I and II in the skeletal-muscle sodium channel may interact only with domain I in a neuronal channel. Furthermore, the specific channel residues that are essential for toxin binding are unique to each paddle-toxin pair, although the effect of a toxin on the channel mostly depends on which paddle it affects and not on the specific residues in the paddle where the interaction occurs.

Second, the way the specific paddle targeted affects the channel is consistent with current understanding of sodium-channel gating. A toxin that binds to the paddles from domains I-III shifts the voltage range over which the channel opens, making the channel less likely to open over their typical voltage range, whereas a toxin that interacts with domain IV interferes with the channel's ability to become inactive. This observation could be relevant for designing drugs that selectively target voltage-sensor paddles to treat conditions such as epilepsy, long QT syndrome type 3 and many forms of periodic paralysis. These disorders are associated with a multitude of biophysical alterations in sodium-channel gating — including shifts in the voltage range for channel activation or defects in its inactivation - and therefore require specific and targeted treatment strategies. So Bosmans and colleagues' model system ${ }^{1}$, in which targeted drugs and toxins can be tested for how they interact with the unique quartet of voltage sensors in sodium channels, not only contributes to our understanding of channel gating, but will also help design new treatments.

More generally, the authors' method can be extended to paddles from other channels including the many other sodium channels, some of which gate quite differently from one another - or to make different sets of chimaeras using other transmembrane segments of sodium-channel domains. What's more, distinct channel variants with subtle differences in gating properties contribute to such diverse physiological functions as sensing pain and controlling muscle contraction. This approach should therefore greatly advance dissection of the molecular components underlying these subtle but physiologically essential functions. 


\section{References}

1. Bosmans F, Martin-Eauclaire MF, Swartz K. Nature 2008;456:202-208. [PubMed: 19005548]

2. Yellen GQ. Rev Biophys 1998;31:239-295.

3. Jiang Y, et al. Nature 2003;423:33-41. [PubMed: 12721618]

4. Jiang Y, et al. Nature 2003;423:42-48. [PubMed: 12721619]

5. Chanda B, Asamoah OK, Bezanilla F. J Gen Physiol 2004;123:217-230. [PubMed: 14981134]

6. Jiang Y, et al. Nature 2003;432:33-41. [PubMed: 12721618] 
a

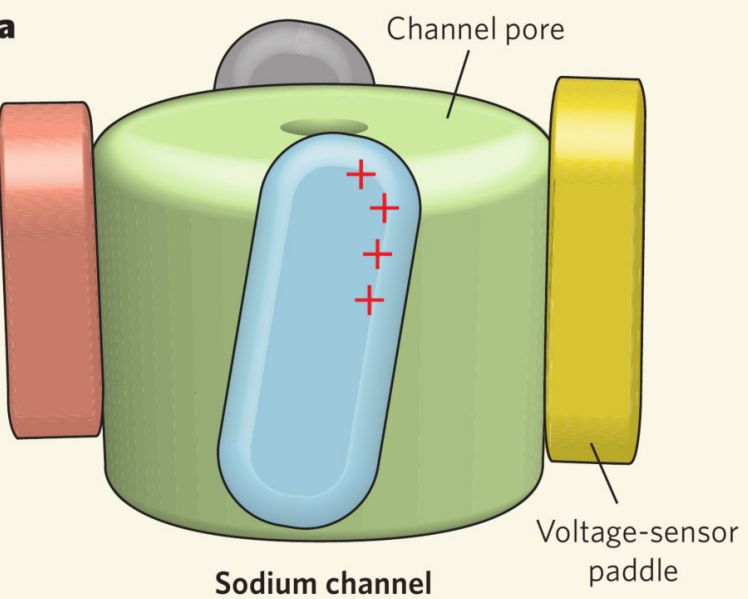

b

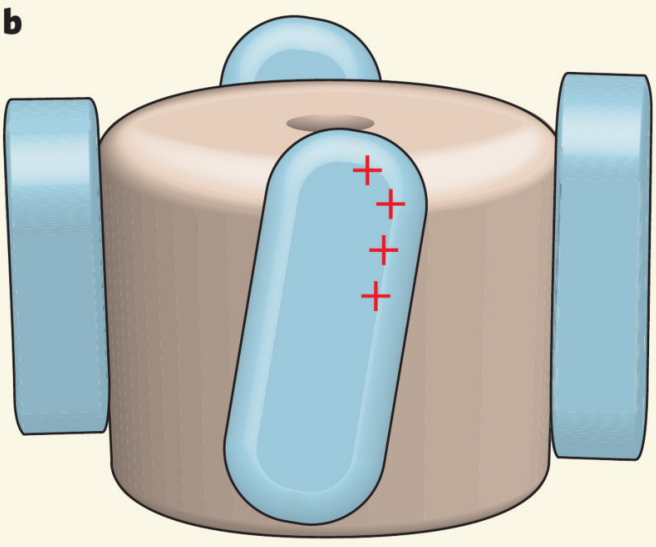

Chimaeric channel

Figure 1. Chimaera channels

a, Studying voltage sensors of sodium channels has been difficult, partly because these channels are made from four domains, each with a unique voltage paddle. b, Bosman et al. ${ }^{1}$ describe a method for looking at voltage sensors of sodium channels in isolation by inserting each paddle in all four positions in a potassium channel. (Adapted from ref. 6 .) 